\title{
Tratamento cirúrgico de fratura do complexo zigomático orbital: relato de caso
}

\author{
Surgical treatment of the zygomatic complex orbital fracture: case report \\ Tratamiento quirúrgico de la fractura del complejo orbitomalar: \\ reporte de caso
}

\author{
Jose Carlos GARCIA DE MENDONÇA ${ }^{1}$ \\ Ellen Cristina GAETTI JARDIM 2 \\ Matheus Augusto dos SANTOS 3 \\ Weckeslley Leonardo Assis XIMENES ${ }^{3}$ \\ Cauê Monteiro dos SANTOS ${ }^{3}$ \\ Diones Calado de QUADROS ${ }^{3}$ \\ Fernando Ribeiro TEIXEIRA ${ }^{3}$ \\ Juliana Andrade MACENA ${ }^{3}$
}

\begin{abstract}
${ }^{I}$ Especialista em Cirurgia e Traumatologia Bucomaxilofacial (CTBMF); Mestre em Ciências da Saúde, pela Universidade de Brasília - UnB; Doutor em Ciências da Saúde (CTBMF), pela Faculdade de Medicina da Universidade Federal do Mato Grosso do Sul-UFMS; Professor Associado da CTBMF da Faculdade de Odontologia - Faodo/UFMS; Coordenador do Programa de Residência em CTBMF do Núcleo de Hospital Universitário "Maria Aparecida Pedrossian" - Universidade Federal do Mato Grosso do Sul, UFMS.

${ }^{2}$ Residência em Cirurgia e Traumatologia Bucomaxilofacial, Hospital Universitário "Maria Aparecida Pedrossian”, Universidade Federal do Mato Grosso do Sul, UFMS, Doutora em Cirurgia e Traumatologia Bucomaxilofacial, Faculdade de Odontologia de Araçatuba, UNESP-Univ. Estadual Paulista, Araçatuba-SP, Brasil. Professora da CTBMF da Faculdade de Odontologia - Faodo/UFMS

${ }^{3}$ Residente em Cirurgia e Traumatologia Bucomaxilofacial (CTBMF) do Núcleo de Hospital Universitário "Maria Aparecida Pedrossian",
\end{abstract} Universidade Federal do Mato Grosso do Sul, UFMS.

\section{Resumo}

As fraturas da região do complexo zigomático orbital envolvem estruturas anatômicas nobres. O diagnóstico dessas fraturas é baseado em achados clínicos, assim como exames complementares por imagem, apresentando papel muito importante, seja para definir a extensão das fraturas, bem como para estabelecer o planejamento do tratamento. No presente trabalho, é apresentado um relato de paciente de 24 anos, gênero masculino, vítima de acidente motociclístico, com trauma de face, apresentando edema aparente em região periocular, equimose periorbitária, hemorragia subconjuntival e afundamento do complexo zigomáticoorbitário esquerdos, causando assimetria facial. O diagnóstico clínico e imaginológico foi de fratura do complexo zigomático orbital a esquerda. Optou-se pelo tratamento cirúrgico com redução e fixação das fraturas mencionadas com bom posicionamento do corpo do osso zigomático, assim como do arco zigomático também fraturado. Num pós-operatório tardio é possível notar a estabilidade do tratamento cirúrgico cruento, seguido de uma boa e quase imperceptível cicatrização dos acessos cirúrgicos aqui preconizados. Descritores: Traumatismos Faciais; Diagnóstico; Terapêutica; Fraturas Orbitárias.

\begin{abstract}
Fractures of the orbital zygomatic complex area involving noble anatomical structures. The diagnosis of these fractures is based on clinical findings, as well as complementary imaging tests, presenting very important role, is to define the extent of fractures, as well as to establish treatment planning. In this study, a patient report of 24 year old male is presented, motorcycle accident victim with facial trauma, with apparent edema periocular, periorbital ecchymosis, subconjunctival hemorrhage and sinking of the left zigomáticoorbitário complex, causing facial asymmetry. The clinical diagnosis was imaginologic and zygomatic orbital complex left fracture. We opted for the surgical treatment with reduction and fixation of fractures mentioned with good body position of the zygomatic bone, as well as the zygomatic arch also fractured. In the late postoperative period it is possible to note the stability of the gory surgical treatment, followed by a good and almost unnoticeable healing of surgical approaches here recommended.
\end{abstract}

Descriptors: Facial Injuries; Diagnosis; Therapeutics; Orbital Fractures.

\section{Resumen}

Las fracturas de la región del complejo orbitomalar envuelven estructuras anatómicas nobles. El diagnóstico de estas fracturas es basado en hallazgos clínicos, así como de imágenes diagnosticas complementarias, que presentan un papel muy importante para definir la extensión de las fracturas, así como para establecer el plan de tratamiento. En este estudio, se presenta un reporte de caso de un paciente de 24 años, sexo masculino, víctima de un accidente de motocicleta, con trauma facial, aparente edema periocular, equimosis periorbitaria, hemorragia subconjuntiva y hundimiento del complejo orbitomalar izquierdo, causando asimetría facial. El diagnóstico clínico y radiográfico fue de fractura del complejo orbitomalar. Se optó por el tratamiento quirúrgico con reducción y fijación de las fracturas mencionadas, con buena posición del cuerpo del hueso cigomático, así como también del arco cigomático fracturado. En el postoperatorio tardío es posible notar la estabilidad del tratamiento quirúrgico cruento, seguido de una buena y casi imperceptible cicatrización de los accesos quirúrgicos aquí recomendados.

Descriptores: Traumatismos Faciales; Diagnóstico; Terapéutica; Fracturas Orbitales. 


\section{INTRODUÇÃO}

O osso zigomático é uma estrutura importante no terço médio de face, mantendo a estética em região malar devido a sua significativa proeminência, e sendo suporte como parede lateral da órbita ${ }^{1}$. Esta estrutura pode ser submetida a diversas forças traumáticas ${ }^{2}$ sendo as regiões que fraturam com maior frequência, as suturas frontozigomático, zigomaticotemporal $^{2,3}$. zigomaticomaxilar

Fatores sócio econômicos, educacionais, governamentais, estão associados com variações epidemiológicas em fraturas do complexo zigomáticoorbital, assim como, mais especificadamente o gênero e idade da vítima, e se tratando de trauma, os acidentes motociclisticos, quedas e agressões físicas predominam esses traumas maxilofaciais ${ }^{1,3,4}$.

Gomes et al. ${ }^{5}$ avaliaram os prontuários de 1.857 pacientes com trauma maxilofacial dos quais 371 apresentavam envolvimento do complexo zigomáticoorbitário. Os pacientes do gênero masculino estavam relacionados com $82,75 \%$ destas fraturas, e em menor quantidade, pacientes do gênero feminino $(17,25 \%)$. A faixa etária compreendia entre 2 a 88 anos, sendo que para os pacientes do gênero masculino a idade variava de 21 a 40 anos 5 .

A grande importância do tratamento cirúrgico das fraturas do complexo zigomático-orbital é restabelecer a proteção ocular e devolver os contornos normais e a proeminência da face, devolvendo estética. O complexo zigomático é responsável pela dissipação de forças sobre o seu corpo e importante transmissor das cargas mastigatórias, sendo fundamental o seu restabelecimento anatômico na face. Fraturas do corpo e arco zigomático podem interferir significantemente na abertura e fechamento bucal e ainda estar relacionado com parestesia do nervo infra-orbital, enoftalmia, diplopia, e limitação na motilidade ocularar, o que configura o tratamento cirúrgico como o tratamento de eleição para as fraturas mencionadas ${ }^{67}$.

O objetivo deste trabalho é apresentar um caso clínico de um paciente de 24 anos de idade, vítima de acidente motociclístico, apresentando fratura do complexo zigomático-orbital tratada cirurgicamente.

\section{CASO CLÍNICO}

Paciente, 24 anos, gênero masculino, vítima de acidente motociclístico, procurou o Serviço de Cirurgia e Traumatologia Bucomaxilofacial do Núcleo de Hospital Universitário da UFMS, com trauma de face, após 01 dia do acidente. Ao exame clínico, apresentava edema $(+1 /+4)$ em região periocular, equimose periorbitária, hemorragia subconjuntival e afundamento do complexo zigomáticoorbitário esquerdo, causando assimetria facial (Figura1). $\mathrm{O}$ paciente apresentava queixas algicas espontâneas e parestesia na região infra-orbitária esquerda, e trismo. À palpação, observou-se degrau na região do rebordo infraorbitário, na sutura frontozigomática e afundamento do arco zigomático esquerdo, assim como o comprometimento do pilar zigomáticomaxilar ipsilateral. Ao exame oftalmológico, a acuidade visual, a motilidade ocular e o reflexo fotomotor estavam preservados, ausência de distopia, diplopia e enoftalmia. Aos exames radiográficos, na incidência de Waters observou-se imagem sugestiva de fratura do rebordo infra-orbitário, sutura fronto-zigomática, discreto velamento do seio maxilar do lado afetado (esquerdo), e ligeira rotação do corpo do zigoma, dificultando a visualização de fratura no pilar zigomático-maxilar (Figura 2). Na incidência radiográfica de Hirtz é nitidamente notório o afundamento do arco zigomático e fratura da sutura Zigomático-Temporal (Figura 3).

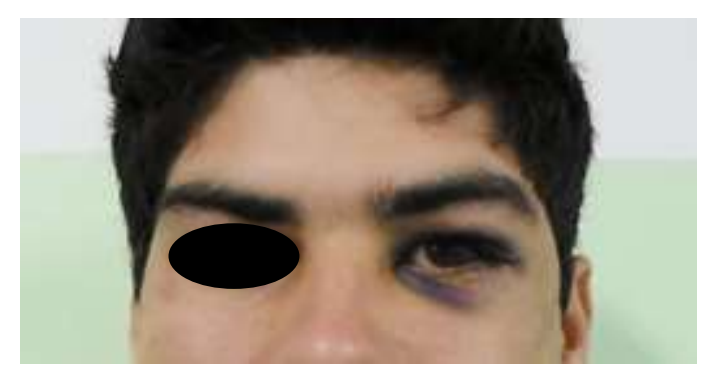

Figura 1. Paciente em norma frontal (Sinais Clínicos). Note equimose periorbitária, hiposfagma em região lateral da esclera, e ligeira oclusão palpebral em região do canto lateral do olho

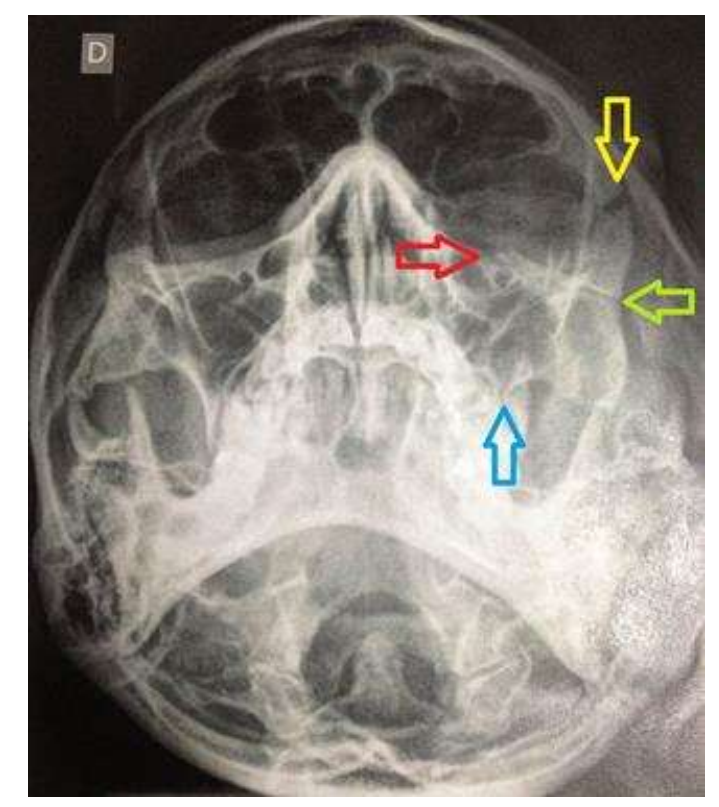

Figura 2. Incidência radiográfica de Waters. Note as regiões de fratura: pilar frontozigomático em dois pontos (seta amarela e verde); margem infra-orbital (seta vermelha); a região do pilar zigomaticomaxilar não se mostra tão nítido devido a sobreposição de imagens (seta azul)

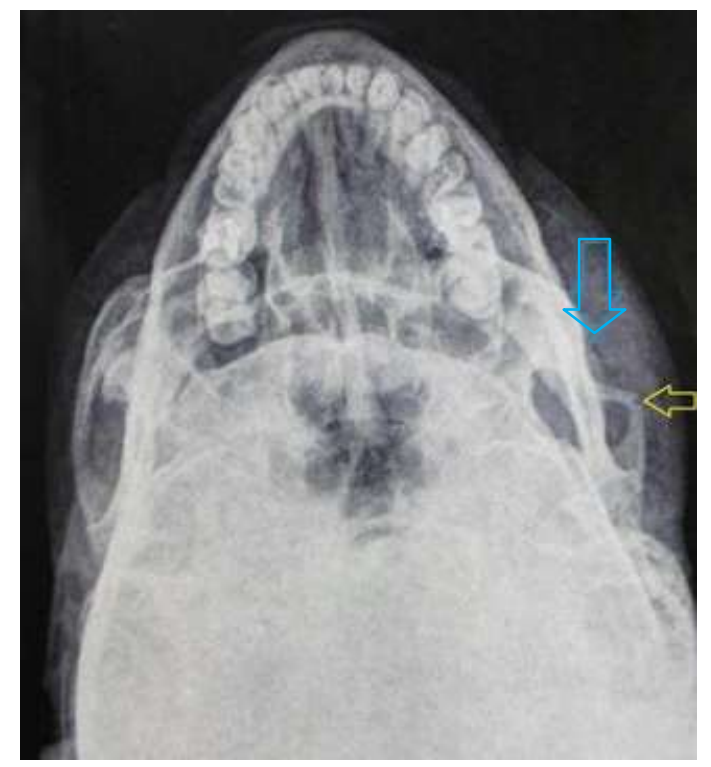

Figura 3. Incidência Radiográfica de Hirtz. Note o afundamento significativo do arco zigomático (seta azul) e fratura na sutura zigomaticotemporal (seta amarela) 
Frente ao exposto e na incapacidade de ação normal do aparelho mastigatório optou-se pelo procedimento cirúrgico de redução e fixação dos pontos fraturados sob anestesia geral, com intubação nasoendotraqueal. Realizou-se assepsia e antissepsia com PVPI 10\% (Polivinilpirrolidona iodo), aposição de campos operatórios e infiltrações com Lidocaína $2 \%$ com epinefrina 1:100.000 (em região periorbitária e em fundo de fornix esquerdo). O globo ocular foi protegido pela tarsorrafia e as fraturas do rebordo infra-orbitário e assoalho da órbita foram expostas através do acesso subtarsal (Figura 4). A fratura da sutura frontozigomática foi exposta através do acesso superciliar (Figura 5). O acesso de Cadwel Luck foi utilizado para acesso ao pilar zigomaticomaxilar (Figura 6).

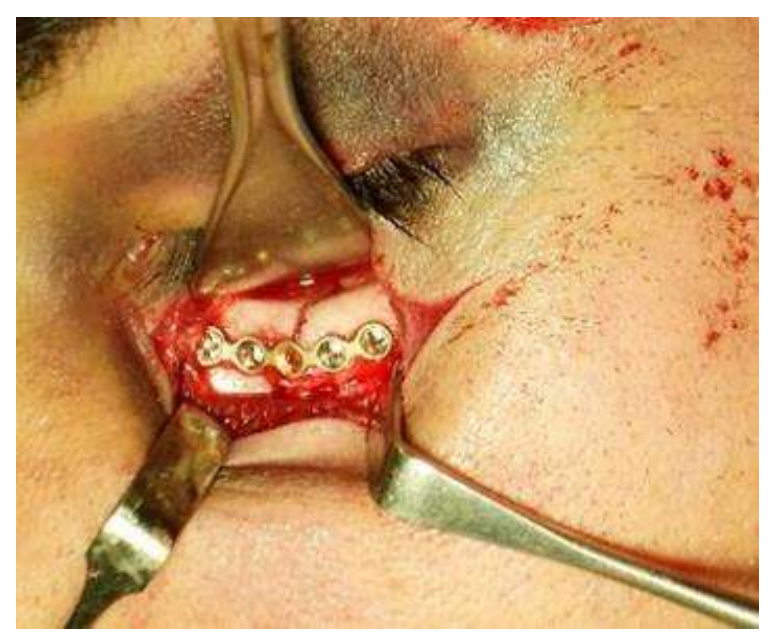

Figura 4. Acesso Subtarsal :rebordo infra-orbitário reduzido e fixado com placas do sistema 2.0

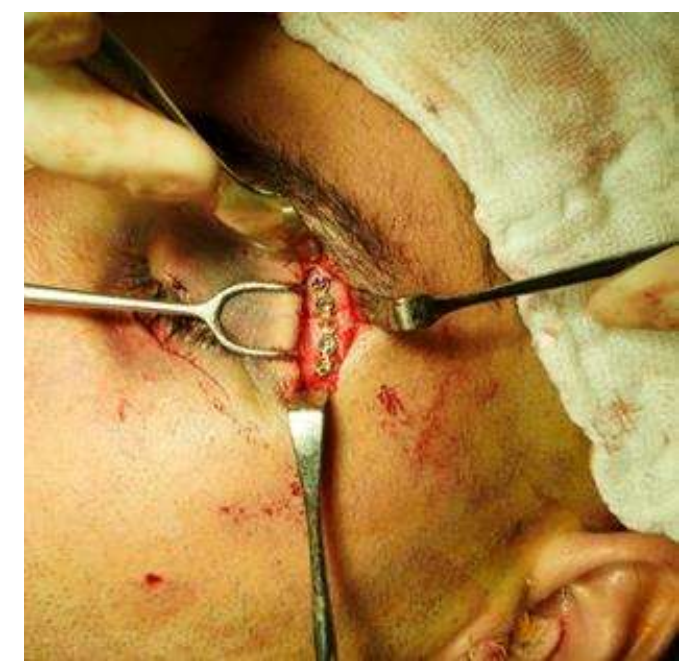

Figura 5. Acesso superciliar: sutura frontozigomática reduzida e fixado com placas do sistema 2.0 (note tarsorrafia)

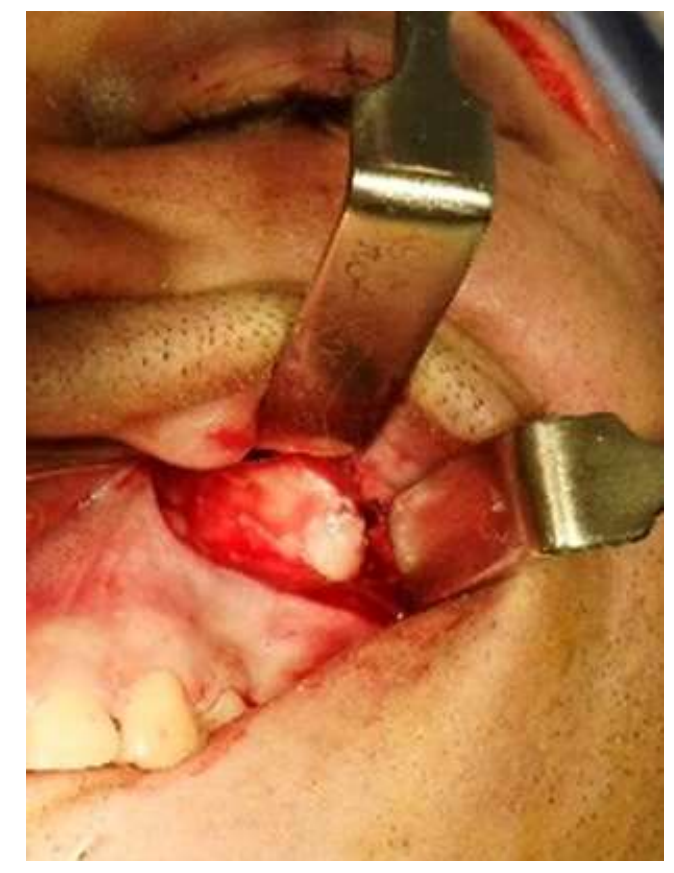

Figura 6. Acesso de Cadwel Luck
As fraturas foram reduzidas com auxílio de gancho de Barros e fixadas com miniplacas e parafusos de titânio do sistema $2.0 \mathrm{~mm}$, o arco zigomático ficou bem posicionado e estável após a redução do corpo do osso zigomático. As suturas por planos foram realizadas com poliglactina 910 , e sutura em pele com fio de nylon 5.0 .

Aos exames radiográficos no pós-operatório imediato, pode ser observado na incidência de Waters que havia satisfatória redução e fixação das fraturas, e na incidência de Hirtz que houve bom reposicionamento conservador do arco zigomático (Figuras 7 e 8).

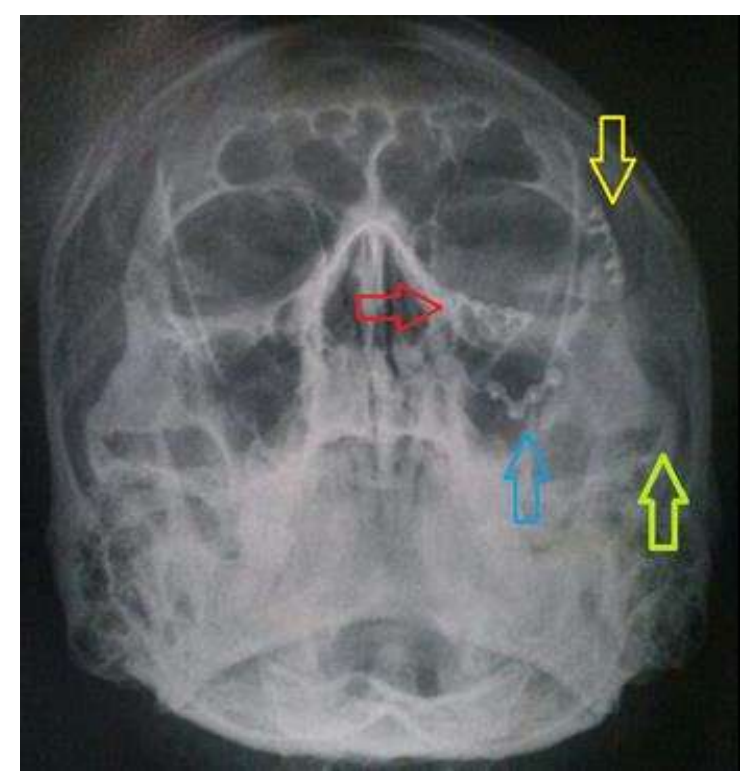

Figura 7. Incidência de Waters no pós-operatório imediato. Note a redução e fixação em três pontos: pilar frontozigomático (seta amarela), margem infra-orbital (seta vermelha) e pilar zigomaticomaxilar (seta azul). Nesta figura é possível notar um bom posicionamento do arco zigomático se comparado com o lado contralateral (seta verde)

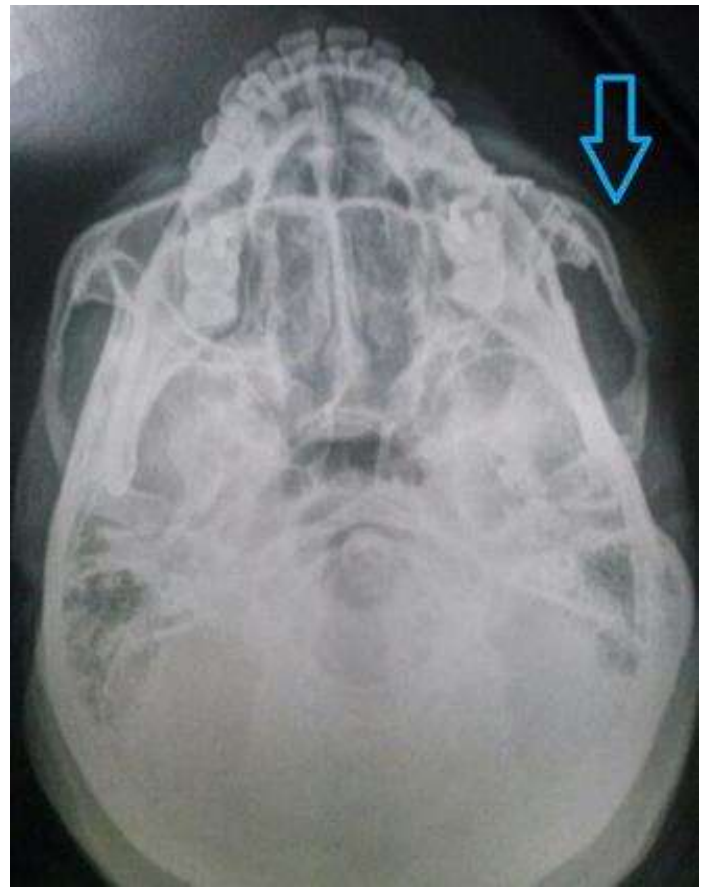

Figura 8. Incidência de Hirtz no pós-operatório imediato. Note o ganho de proeminência malar após redução e fixação do corpo do zigoma (seta azul)

No pós-operatório de 10 dias o paciente apresentava excelente cicatrização dos tecidos, com edema sutil em face esquerda, apresentando sinais da hemorragia subconjuntival. Durante todo o controle pósoperatório, o paciente apresentava-se sem sinais de infecção. A cicatrização das feridas encontrava-se em bom processo de reparo e a projeção zigomática esquerda restabelecida. Os movimentos oculares se mostravam preservados, com amplitude normal, sem 
nenhuma complicação oftalmológica. A abertura bucal apresentou-se sem limitações em sua amplitude (Figura 9). No pós-operatório de 40 dias, houve uma melhora significativa nas cicatrizes do acesso subtarsal e superciliar (Figura 10).

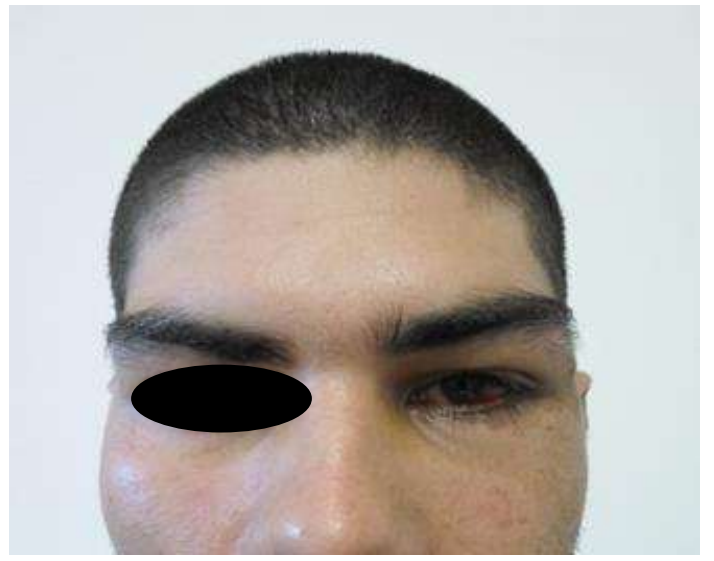

Figura 9. Pós-operatório de 10 dias. Note a melhora significativa da hiposfagma, pequenos sinais de equimose periorbital, pálpebras ainda ligeiramente ocluídas em canto lateral

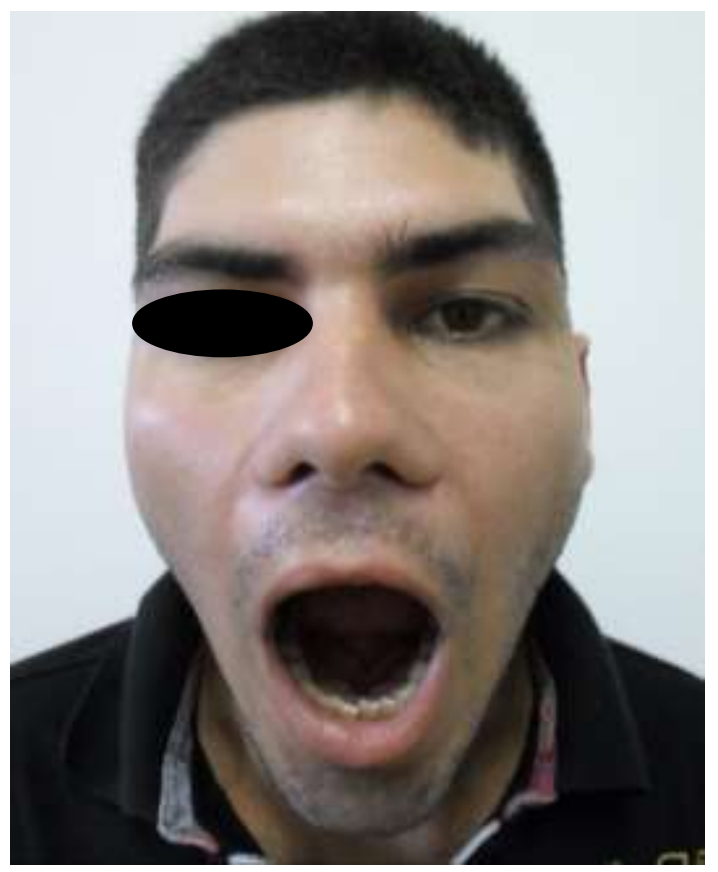

Figura 10. Pós-operatório de 40 dias.

É possível notar melhora significativa nos sinais anteriormente descritos nas Figuras 1 e 9 . Note a abertura bucal sem limitações funcionais

Mais tardiamente, com 90 dias após a cirurgia, as cicatrizes ficaram praticamente imperceptíveis. O paciente apresentou ótima recuperação durante a avaliação do curso clínico pós-operatório (Figura 11).

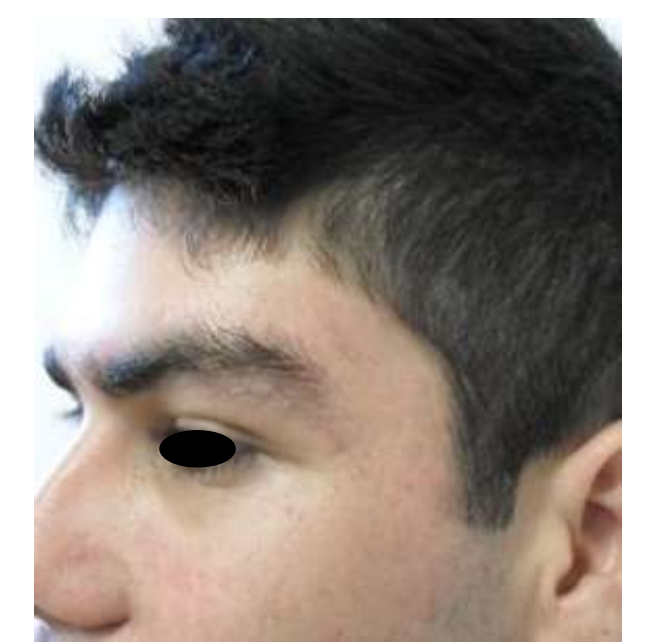

Figura 11. Pós-operatório de 90 dias. Neste observamos o aspecto quase que imperceptível da cicatriz do acesso sub-tarsal, o acesso superciliar mostra-se com aspecto satisfatório. A proeminência malar é mantida

\section{DISCUSSÃO}

Fraturas múltiplas da face sobretudo que envolvam o arcabouço orbitário apresentam uma variedade de métodos de tratamento, conservadores ou cirúrgicos ${ }^{89}$. O tratamento conservador está indicado para fraturas com mínimo e/ou sem deslocamento ${ }^{10}$. $\mathrm{O}$ tratamento cirúrgico das fraturas do complexo zigomático orbital e arco zigomático estão indicados quando há presença de fraturas com deslocamentos significativos, associados a limitação da abertura bucal, comprometimento da motilidade ocular e alterações na acuidade visual, assim como defeitos estéticos na face ${ }^{1112}$.

No presente caso, o paciente referido apresentou deslocamento significativo do arco zigomático o qual limitava a abertura bucal. Degraus ósseos em região de pilar froto-zigomático, margem infra orbital e pilar zigomático-maxilar foram nitidamente notados ao exame físico os membros corroborados em tomadas radiográficas. Deste modo o tratamento cirúrgico cruento com redução e fixação interna rígida foi à opção de escolha.

No tratamento cirúrgico alguns acessos foram considerados para os pilares comprometidos (Pilar Fronto-Zigomático, Zigomático-Maxilar, e o horinzontal, moldura Infra-Orbital). Para região de rebordo infraorbital, o acesso de eleição foi o sub-tarsal, devido aos menores índices de complicações e facilidade de técnica, se comparado ao acesso sub-ciliar ou transconjultival.

A escolha do acesso sub-tarsal, se deu em virtude de seu menor índice de retração palpebral, consequentemente menor exposição da esclera no pósoperatório tardio, e menor índice de ectrópio cicatricial quando comparado ao acesso sub-ciliar. ${ }^{1314}{ }^{15}$.

O paciente referido neste trabalho apresentava linhas de expressões favoráveis em região palpebral inferior, o que foi "ponto chave" para a eleição do acesso, resultando no pós-operatório tardio um aspecto estético, sendo praticamente imperceptível a linha de incisão utilizado neste caso.

Para o pilar frontozigomático o acesso de escolha foi o superciliar. Consideramos o gênero do paciente, o limite do supercílio, e a facilidade do acesso, o que trouxe um bom resultado no último registro do pósoperatório deste paciente. A abordagem do pilar zigomaticomaxilar foi realizada por meio de acesso intra-bucal de Cadwel Luck com extensão na região posterior, optamos pelo "bisturi frio" nesta técnica, onde ofereceu uma menor redução do fundo de vestíbulo.

Uma variedade de tratamentos para o complexo zigomático vem sendo citado após o primeiro relato de redução cirúrgica de Duverney ${ }^{16}$.

Neste relato de caso a técnica de redução do complexo zigomático foi percutâneo, com o auxílio do Gancho de Barros. Houve um bom posicionamento da estrutura do corpo do zigoma, influenciando na redução conservadora do arco zigomático.

Alguns autores citam a fixação em três pontos sendo indicada quando a fratura se encontra instável ${ }^{8,9}$. Optamos por realizar a osteossíntese com placas e 
parafusos de fixação interna rígida seguindo este padrão citado, em três pontos. (margem infra-orbital, pilar frontozigomático, e pilar zigomaticomaxilar).

No presente caso, a técnica trouxe um bom resultado, inclusive para maior estabilidade do arco zigomático comprometido no trauma. A síntese tecidual foi realizada por planos, com a utilização de poliglactina 9104.0 violeta nos tecidos profundos, e superficialmente em pele com nylon 5.0. Obtivemos bons resultados pósoperatórios.

\section{CONCLUSÃO}

O bom posicionamento das fraturas abordadas proporcionou uma correta função nos movimentos de abertura e fechamento bucal além da correta projeção ocular e estética facial. Em traumas faciais, o importante a ser ressaltado é a individualização de cada caso, para oferecer o melhor tratamento possível levando em consideração os recursos e tratamentos disponíveis atualmente. Até o momento pode-se considerar sucesso estético e funcional ao caso apresentado.

\section{REFERÊNCIAS}

1. Ellis E III. Fractures of the zygomatic complex and arch. In: Fonseca RJ, Walker RV, Betts NJ, Barber HD, editors. Oral and maxillofacial trauma. Philadelphia: W.B. Saunders; 1997. p. 571-632.

2. Ugboko V, Udoye C, Ndukwe K, Amole A, Aregbesola S. Zygomatic complex fractures in a suburban Nigerian population. Dent Traumatol 2005 Apr; 21(2):70-5.

3. Brasileiro BF, Passeri LA. Epidemiological analysis of maxillofacial fractures in Brazil: a 5-year prospective study. Oral Surg Oral Med Oral Pathol Oral Radiol Endod 2006 Jul; 102(1): 28-34.

4. Hogg NJV, Stewart TC, Armstrong JE, Girotti MJ. Epidemiology of maxillofacial injuries at trauma hospitals in Ontario, Canada, between 1992 and 1997. J Trauma 2000 Sep; 49(3):425-32.

5. Gomes PP, Passeri LA, Barbosa JR. A 5-year retrospective study of zygomatico-orbital complex and zygomatic arch fractures in Sao Paulo State, Brazil. J Oral Maxillofac Surg. 2006 Jan; 64(1):63-7.

6. Kloss FR, Stigler RG, Brandstätter A, Tuli T, Rasse $\mathrm{M}$, Laimer $\mathrm{K}$, et al. Complications related to midfacial fractures: operative versus non-surgical treatment. Int J Oral Maxillofac Surg 2011 Jan;40(1):33-7.

7. Kurita M, Okazaki M, Ozaki M, Tanaka Y, Tsuji N, Takushima A, et al. Patient satisfaction after open reduction and internal fixation of zygomatic bone fractures. J Craniofac Surg 2010 Jan; 21(7):45-9.

8. Lee EI, Mohan K, Koshy JC, Hollier LH Jr. Optimizing the surgical management of zygomaticomaxillary complex fractures. Semin Plast Surg 2010 Nov;24(4):389-97.

9. Kelley P, Hopper R, Gruss J. Evaluation and treatment of zygomatic fractures. Plast Reconstr Surg 2007 Dec; 120(7 Suppl 2):5S-15S.

10. Evans BG, Evans GR. MOC-PSSM CME article: zygomatic fractures. Plast Reconstr Surg 2008 Jan; 121(1 Suppl):1-11.

11. Hollier LH, Thornton J, Pazmino P, Stal S. The management of orbitozygomatic fractures. Plast Reconstr Surg 2003 Jun;111(7):2386-92, quiz 2393.

12. Zingg M, Laedrach K, Chen J, Chowdhury K, Vuillemin T, Sutter F, et al. Classification and treatment of zygomatic fractures: a review of 1,025 cases. J OralMaxillofac Surg 1992 Aug; 50(8):778-90.

13. Ellis E, Zide MF. Periorbital approaches. In: Ellis E, Zide MF, editors. Surgical access to the facial skeleton. Baltimore (MD): Williams \& Wilkins; 1995. p. 7-54.

14. Kushner GM. Surgical approaches to the infraorbital rim and orbital floor: the case for the transconjunctival approach. J Oral Maxillofac Surg 2006 Jan; 64(1):108-10.

15. Khan AM, Varvares MA. Traditional approaches to the orbit. Otolaryngol Clin North Am 2006 Oct;39(5): 895-909.

16. Carter TG, Bagheri S, Dierks EJ. Towel Clip Reduction of the Depressed Zygomatic Arch Fracture. J Oral Maxillofac Surg. 2005 Aug; 63(8):1244-6.

\section{CONFLITO DE INTERESSES}

Os autores declaram não haver conflitos de interesse.

\section{AUTOR PARA CORRESPONDÊNCIA}

Ellen Cristina Gaetti Jardim

ellengaetti@gmail.com.com

Submetido em 11/08/2016

Aceito em 16/08/2016 